
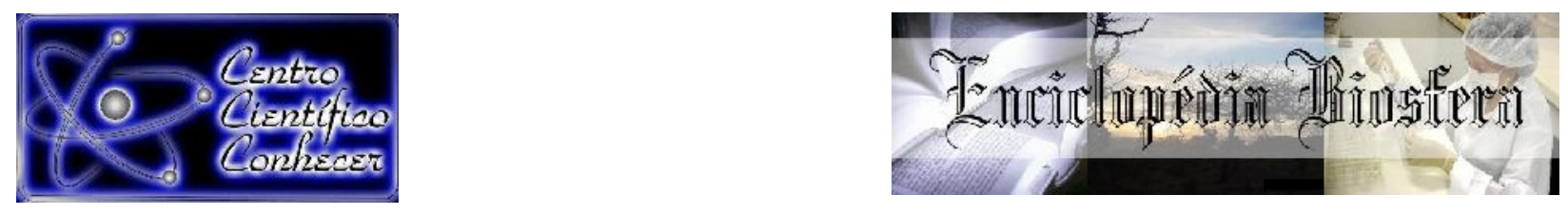

\title{
AVALIAÇÃO DO POTENCIAL ANTIOXIDANTE DO ÓLEO ESSENCIAL DE Thymus vulgaris L. (Lamiaceae)
}

\author{
Mateus Sousa Porto ${ }^{1}$, Yanes Neves Valadares ${ }^{2}$, Cláudia do Nascimento \\ Silva $^{2}$, Rafaela Brito Ribeiro Santos ${ }^{2}$, Simone Andrade Gualberto ${ }^{3}$ \\ ${ }^{1}$ Graduando em Química com Atribuições Tecnológicas pela Universidade \\ estadual do Sudoeste da Bahia (UESB), Campus Juvino Oliveira, Itapetinga- \\ BA. \\ ${ }^{2}$ Graduanda em Ciências Biológicas pela Universidade do Estado do \\ Sudoeste da Bahia (UESB), Campus Juvino Oliveira, Itapetinga-BA. \\ ${ }^{3}$ Professora. Dra. Laboratório de Pesquisas em Produtos Naturais - \\ Departamento de Ciências Exatas e Naturais - Universidade Estadual do \\ Sudoeste da Bahia - Itapetinga, Brasil. \\ E-mail: mateus.sp95@hotmail.com
}

\section{Recebido em: 15/11/2021 - Aprovado em: 15/12/2021 - Publicado em: 30/12/2021 DOI: 10.18677/EnciBio_2021D8

\section{RESUMO}

Os óleos essenciais têm se tornado foco de estudos e interesse da comunidade científica, principalmente na área farmacêutica, onde a busca por fitoterápicos de ampla utilidade torna-se crucial. Muitas das espécies vegetais endêmicas e exóticas são plantas medicinais produtoras de óleos essenciais. Essas espécies têm contribuído de maneira importante para as indústrias, principalmente como aditivos em alimentos e em aplicações terapêuticas. Essa pesquisa teve por objetivo realizar a avaliação da da atividade antioxidante do óleo essencial comercial da espécie Thymus vulgaris L., popularmente conhecida como tomilho e verificar a correlação com sua composição química. O óleo essencial foi adquirido da empresa Phytoterápica - Solua Comercial Eireli - Epp. O potencial antioxidante do óleo essencial foi avaliado pelo método da redução do radical livre DPPH (2,2-difenil-1-picril-hidrazil) e pelo poder redutor de íons ferro - Frap (Ferric Power Reducing). A atividade antioxidante, determinada pelo método do DPPH, foi expressa em concentração efetiva $50\left(\mathrm{CE}_{50}\right)$, correspondente a 908,07 $\pm 0,08$ $\mu \mathrm{g} \cdot \mathrm{mL}^{-1}$ e pelo índice de atividade antioxidante $(\mathrm{IAA}=0,03)$. Pelo método do poder redutor de íons ferro (FRAP), o valor obtido foi de 0,344 $\pm 0,157$ miligramas de íons ferro $\left(\mathrm{Fe}^{2+}\right)$ por grama de amostra. Observou-se uma fraca atividade antioxidante para o óleo, provavelmente relacionada ao timol e ao carvacrol, os constituintes majoritários presentes na amostra.

PALAVRAS-CHAVE: Atividades biológicas, Óleos essenciais, Plantas medicinais. 


\title{
EVALUATION OF THE ANTIOXIDANT POTENTIAL OF THE ESSENTIAL OIL OF Thymus vulgaris (Thyme)
}

\begin{abstract}
Essential oils have become the focus of studies and interest in the scientific community, especially in the pharmaceutical area, where the search for widely useful herbal medicines becomes crucial. Many of the endemic and exotic plant species are medicinal plants that produce essential oils. These species have made an important contribution to industries, mainly as food additives and in therapeutic applications. This research aimed to evaluate the antioxidant activity of the commercial essential oil of the species Thymus vulgaris L., popularly known as thyme, and to verify the correlation with its chemical composition. The essential oil was purchased from the company Phytoterápica - Solua Comercial Eireli - Epp. The antioxidant potential of the essential oil was evaluated by the DPPH free radical reduction method (2,2-diphenyl-1-picryl-hydrazyl) and by the reducing power of iron ions - Frap (Ferric Power Reducing). The antioxidant activity, determined by the DPPH method, was expressed in effective concentration 50 (EC50), corresponding to $908.07 \pm 0.08 \mu \mathrm{g} \cdot \mathrm{mL}-1$ and by the antioxidant activity index $(I A A=0.03)$. Using the reducing power of iron ions (FRAP) method, the value obtained was $0.344 \pm 0.157$ milligrams of iron ions ( $\mathrm{Fe} 2+$ ) per gram of sample. There was a weak antioxidant activity for the oil, probably related to thymol and carvacrol, the major constituents present in the sample.
\end{abstract}

KEYWORDS:, Biological activity, Essential oils, Medicinal plants.

\section{INTRODUÇÃO}

A demanda por produtos naturais é crescente, seja por uma questão cultural, pela busca em minimizar ou até erradicar problemas ocasionados pelo uso indiscriminado de produtos químicos sintéticos, ou pelo acesso limitado de determinadas populações a recursos terapêuticos, surgindo a necessidade de se utilizar chás e infusões à base de plantas como alternativas para o tratamento de doenças. Segundo Koehn e Carter (2005), cerca de 30\% das drogas prescritas no mundo são obtidas direta ou indiretamente das plantas. Dentre os produtos obtidos de espécies vegetais e utilizados pela população estão os óleos essenciais.

Os óleos essenciais possuem uma posição de destaque na fitoterapia, devido às suas propriedades farmacológicas, que são reconhecidas pelos povos antigos e muito utilizadas na medicina popular. São compostos líquidos, orgânicos, voláteis, com aromas característicos e lipofílicos, extraídos de diferentes partes da planta, geralmente por destilação por arraste de vapor (NOGUEIRA et al., 2013).

Dentre as propriedades farmacológicas apresentadas pelo óleo essencial da espécie T. vulgaris destacam-se as atividades antioxidante e antimicrobiana, relacionadas à presença de compostos fenólicos . Diante do exposto, essa pesquisa teve por objetivo realizar a avaliação da atividade antioxidante do óleo essencial comercial da espécie Thymus vulgaris, popularmente conhecida como tomilho e verificar a correlação com sua composição química.

\section{MATERIAL E MÉTODOS Aquisição do Óleo Essencial de tomilho}

O óleo essencial da espécie Thymus vulgaris foi obtido comercialmente da empresa Phytoterápica ${ }^{\circledR}$, que concedeu o laudo técnico da composição química do produto com especificações. 


\section{Avaliação da Atividade Antioxidante pelo Método DPPH}

O potencial antioxidante do óleo essencial de Thymus vulgaris $L$. foi avaliado pelo método DPPH descrito por Rufino et al. (2007), utilizando-se etanol ao invés de acetona para melhor solubilização do óleo essencial. O método DPPH consiste na captura do radical livre 2,2-difenil-1- picril-hidrazina (DPPH) por substâncias antioxidantes.

O óleo essencial foi solubilizado em etanol para a obtenção de soluções com as concentrações de 10; 20; 30; 40 e 50 mg. $\mathrm{mL}^{-1}$. Em seguida, em ambiente escuro, $100 \mu \mathrm{L}$ de cada solução foi transferida para tubos âmbar e adicionados $3,9 \mathrm{~mL}$ da solução de DPPH a $0,06 \mathrm{mM}$. Os tubos foram agitados em agitador mecânico (Modelo Vórtex) e, em seguida, foram armazenados em local abrigado da luz por 60 minutos, conforme determinado pelos ensaios de cinética realizados previamente. Padrões de quercetina e rutina foram utilizados como controles positivos, nas concentrações de 25; 50; 75; 100 e $125 \mu \mathrm{g} \cdot \mathrm{mL}^{-1}$ para quercetina e $25 ; 75 ; 125 ; 175$ e $225 \mu \mathrm{g} . \mathrm{mL}^{-1}$ para rutina.

Após este período, realizou-se a leitura das absorbâncias das amostras em espectrofotômetro Shimadzu SPECTROPHOTOMETER UV $-1800^{\circledR}$ no comprimento de onda de $515 \mathrm{~nm}$. Utilizou-se etanol como "branco" para zerar o equipamento. O valor médio das absorbâncias obtidas das leituras das amostras foram relacionados com suas respectivas concentrações e plotadas em um gráfico para a obtenção de uma equação de regressão linear, para encontrar-se o valor da concentração efetiva $\left(\mathrm{CE}_{50}\right)$ do óleo, que corresponde à quantidade de amostra necessária para reduzir a concentração inicial do reagente DPPH em 50\% (SILVA et al., 2017).

Partindo-se da divisão da concentração inicial da solução de DPPH a 0,06 $\mathrm{mM}\left(24 \mu \mathrm{g} \cdot \mathrm{mL}^{-1}\right)$ pelo valor da concentração efetiva $\left(\mathrm{CE}_{50}\right)$ encontrada para a amostra, calculou-se o índice de atividade antioxidante (IAA).

\section{Avaliação da Atividade Antioxidante pelo Método FRAP}

O método FRAP (do inglês, Ferric Reducing Antioxidant Power) é um método de determinação da atividade antioxidante que consiste na redução dos íons férricos presentes no reagente a íons ferrosos, pela ação de compostos redutores presentes na amostra. O potencial antioxidante do óleo essencial comercial do tomilho foi avaliado neste trabalho pela metodologia proposta por Rufino et al. (2006).

Em ambiente escuro, foram adicionados $90 \mu \mathrm{L}$ da solução etanólica do óleo essencial do tomilho (30 mg. $\mathrm{mL}^{-1}$ ) em um tubo de ensaio âmbar e, em seguida, adicionados 2,7 $\mathrm{mL}$ do reagente Frap e $270 \mu \mathrm{L}$ de água deionizada. Após serem agitados em vórtex para homogeneização, os tubos foram colocados em banhomaria a temperatura de $37 \pm 2^{\circ} \mathrm{C}$ por 30 minutos. Em seguida, foram realizadas as leituras das amostras em espectrofotômetro Shimadzu SPECTROPHOTOMETER UV $-1800^{\circledR}$ em comprimento de onda de $595 \mathrm{~nm}$. As amostras foram feitas em triplicata.

Para a construção da curva de regressão linear foram preparadas soluções de sulfato ferroso nas concentrações de 500, 1000, 1500 e $2000 \mu \mathrm{M}$ e submetidas às mesmas condições descritas anteriormente. Os valores médios das leituras das absorbâncias foram plotados no eixo $Y$ e os valores das concentrações das soluções de sulfato ferroso no eixo $X$, utilizando-se o Software Excell. Obteve-se uma equação da reta correspondente a $y=0,0004 x-0,0098$ e uma curva de regressão linear com o coeficiente de correlação de Pearson de 0,998 . A partir da equação da reta da curva analítica realizou-se o cálculo para se obter a concentração em $\mu \mathrm{M}$ de sulfato de ferro heptahidratado, que foi convertido em $\mathrm{mg}$ de íons $\mathrm{Fe}^{2+}$ presentes em 100 gramas de amostra. 


\section{RESULTADOS E DISCUSSÃO}

O resultado da análise cromatográfica mostrou que foram identificados 10 constituintes químicos, correspondente a $97,3 \%$ do total dos constituintes. Os compostos encontrados foram Timol (53,5\%), p-cimeno (20,8\%), Carvacrol (8,9\%), Linalol (8\%), Mirceno (1,6\%), $\alpha$-terpineol $(1,6 \%)$, Desidrocarveol $(1,2 \%)$, Limoneno $(0,8 \%)$, Canfeno $(0,7 \%), \alpha$-pineno $(0,4 \%)$ e outros $(2,7 \%)$.

O óleo essencial de tomilho apresentou valor de $\mathrm{CE}_{50}$ de 908,07 $\pm 0,08 \mu \mathrm{g}$. $\mathrm{mL}^{-1}$, e os controles positivos rutina $\left(\mathrm{CE}_{50} 4,29 \pm 0,08 \mathrm{mg} \mathrm{mL}^{-1}\right.$ ) e quercetina (CE $\left.1,57 \pm 0,13 \mathrm{mg} \cdot \mathrm{mL}^{-1}\right)$. De acordo com esses resultados, o óleo essencial de tomilho apresentou uma baixa capacidade antioxidante em comparação aos controles positivos rutina e quercetina.

O Índice de Atividade Antioxidante (IAA) foi calculado pela divisão do valor de $\mathrm{CE}_{50}$ encontrado para as amostras pela concentração inicial do DPPH 0,06 mM (24 $\mu \mathrm{g} \cdot \mathrm{mL}^{-1}$ ), obtendo-se o valor de IAA= 0,03 para o óleo essencial. Os valores de IAA para a rutina $(I A A=5,59)$ e quercetina $(I A A=15,32)$ também foram calculados (Tabela 1). Scherer e Godoy (2009) propuseram faixas de valores para classificar extratos e óleos essenciais quanto ao poder antioxidante. Amostras consideradas com baixa atividade antioxidante apresentam valores de IAA na faixa de 0,5 a 1,0; com moderada atividade antioxidante na faixa de 1,0 a 2,0 e com atividade antioxidante muito forte acima de 2,0. De acordo com essa avaliação, o óleo essencial analisado apresentou fraca atividade antioxidante nos ensaios realizados. A avaliação quantitativa da atividade antioxidante também foi determinada pelo método FRAP, apresentando o valor de 0,344 $\pm 0,157$ miligramas de íons ferro $\left(\mathrm{Fe}^{2+}\right)$ por grama de amostra.

TABELA 1. Resultados dos testes de avaliação da atividade antioxidante do óleo essencial de tomilho com seus respectivos desvios padrão

\begin{tabular}{llcc}
\hline \multicolumn{1}{c}{ Amostra } & $\begin{array}{c}\text { DPPH } \\
\mathbf{C E}_{50}\left(\mathbf{m g} \cdot \mathbf{m L}^{-1}\right)\end{array}$ & IAA & $\begin{array}{c}\mathrm{FRAP}^{-1} \\
\left(\mathbf{m g ~ F e} \cdot \mathbf{~ g}^{-1} \mathbf{d e}\right. \\
\mathbf{a m o s t r a})\end{array}$ \\
\hline Óleo essencial & $908,07 \pm 0,08$ & 0,03 & $0,344 \pm 0,157$ \\
Rutina & $4,29 \pm 0,08$ & 5,59 & - \\
Quercetina & $1,57 \pm 0,13$ & 15,32 & - \\
\hline
\end{tabular}

IAA (índice de atividade antioxidante); DPPH (2,2-difenil-1-picrilhidrazina); FRAP (Ferric

Reducing Antioxidant Power); (-) Não avaliado

Crespo et al. (2019) encontraram uma alta atividade antioxidante para o óleo essencial de tomilho, expressa em porcetagem de inibição do radical livre DPPH de até $67 \%$, diferente deste trabalho. Satyal et al. (2016) e Khan et al. (2019), ao desenvolverem trabalhos semelhantes, constataram a influência que a espécie Thymus vulgaris sofre ao se desenvolver em diferentes regiões geográficas, encontrando grandes alterações na produção dos metabólicos secundários timol, linalol, geraniol, $p$-cimeno, $y$-terpineno, limoneno, borneol e carvacrol nas espécies estudadas. Também confirmaram a presença de compostos diferentes entre coletas, como o composto acetato de geranila. 
Fatores bióticos e abióticos podem alterar as rotas biossintéticas nas espécies vegetais, levando à síntese de metabólitos secundários diversos, em resposta aos estresses a que são expostas (KHALIL et al., 2018). Por consequência, essas interferências podem contribuir para o acúmulo de metabólitos secundários nas plantas, ocasionando em propriedades biológicas distintas.

O estudo desenvolvido com o óleo essencial da Thymus vulgaris por Kulisic et al. (2005) mostrou que, a depender das classes de metabólitos secundários contidos na espécie, esta apresenta uma baixa atividade antioxidante e atribui esta baixa atividade à predominância de compostos terpênicos frente aos compostos fenólicos no óleo. Gedikoğlu e Sökmen (2019) concluíram que o timol e o carvacrol são os compostos responsáveis pela atividade antioxidante do óleo essencial de tomilho, colaborando para o entendimento de que a capacidade antioxidante destes óleos está relacionada à concentração destes compostos nas amostras.

A variação entre os teores de timol e carvacrol presentes nos óleos essenciais pode afetar o potencial antioxidante das espécie do gênero Thymus (MANCINI et al., 2015) e essa variação pode ser explicada pela relação biosintética entre os dois compostos. A síntese do timol juntamente com a do carvacrol, ocorre pela via metabólica de formação do $\gamma$-terpineno, onde este último é oxidado a $p$-cimeno e, em seguida, sofre hidroxilação gerando como produtos o timol e o carvacrol (WESOLOWSKA ; JADCZAK, 2019). As vias metabólicas ativadas nas espécies vegetais proporcionam a síntese de vários compostos, dependendo das necessidades das mesmas.

Há relatos na literatura de seis diferentes quimiotipos de Thymus vulgaris, com produção de diferentes óleos essenciais, quimiotipos fenólicos (timol e carvacrol), quimiotipos não fenólicos (geraniol, $\alpha$-terpineol, linalol e trans-4-terpineol), com diferentes atividades biológicas entre os quimiotipos do mesmo grupo ou de grupos diferentes (TORRAS et al., 2007).

\section{CONCLUSÃO}

Verificou-se que os constituintes majoritáios presentes no óleo essencial do tomilho analisado são o timol (53,3\%), o carvacrol $(8,9 \%)$ e p-cimeno $(20,8 \%)$. O óleo essencial da Thymus vulgaris comercial analisado apresentou uma baixa capacidade antioxidante, possivelmente devido à sua composição de metabólitos secundários.

\section{REFERÊNCIAS}

CRESPO, Y. A. SÁNCHEZ, L. R. B.; QUINTANA, Y. G.; CABRERA, A. S. T. et al. Evaluation of the synergistic effects of antioxidant activity on mixtures of the essential oil from Apium graveolens L., Thymus vulgaris L. and Coriandrum sativum L. using simplex-lattice design. Heliyon, v. 5, n. 6, p. e01942, 2019. Disponivel em:< https://doi.org/10.1016/j.heliyon.2019.e01942>. DOI:10.1016/j.heliyon.2019.e01942.

GEDIKOĞLU, A.; SÖKMEN, M.; ÇIVIT, A. Evaluation of Thymus vulgaris and Thymbra spicata essential oils and plant extracts for chemical composition, antioxidant, and antimicrobial properties. Food Science \& Nutrition, v. 7, n. 5, p. 1704-1714, 2019. Disponível em:<https://doi.org/10.1002/fsn3.1007>. DOI: 10.1002/fsn3.1007.

KHALIL, N.; FEKRY, M.; BISHR, M.; ZALABANI, S.; SALAMA, O. Foliar spraying of salicylic acid induced accumulation of phenolics, increased radical scavenging 
activity and modified the composition of the essential oil of water stressed Thymus vulgaris L. Plant Physiology and Biochemistry, v. 123, p. 65-74, 1 fev. 2018. Disponivel:<https://doi.org/10.1016/j.plaphy.2017.12.007>. 10.1016/j.plaphy.2017.12.007.

KHAN, A. A.; AMJAD, M. S.; SABOON. GC-MS Analysis and biological activities of Thymus vulgaris and Mentha arvensis essential oil. Turkish Journal of Biochemistry, v. 44, n. 3, p. 388-396, 2019. Disponivel:<https://doi.org/10.1515/tjb2018-0258>. DOI:10.1515/tjb-2018-0258.

KOEHN, F.E.;CARTER GT. The evolving role of natural products in drug discovery. Nat Revista Drug Discovery. 2005 Mar;4(3):206-20. Disponível em: < https://doi.org/10.1038/nrd1657 >. DOI: 10.1038/nrd1657.

KULISIC, T.; RADONIC, A.; MILOS, M. Antioxidant properties of thyme (Thymus vulgaris L.) and wild thyme (Thymus serpyllum L.) essential oils. Italian Journal of Food Science, v. 17, n. 3, p. 315-324, 2005. Disponível em: https://www.researchgate.net/publication/287954724_Antioxidant_properties_of_thy me_Thymus_vulgaris_L_and_wild_thyme_Thymus_serpyllum_L_essential_oils.

MANCINI, E.; SENATORE, F.; MONTE, D,D.; MARTINO, L,D.. Studies on Chemical omposition, Antimicrobial and Antioxidant Activities of Five Thymus vulgaris L. Essential Oils. Molecules, v. 20, n. 7, p. 12016-12028, 2015. Disponível:<https://www.researchgate.net/publication/280030242_Studies_on_Chem ical_Composition_Antimicrobial_and_Antioxidant_Activities_of_Five_Thymus_vulgari s_L_Essential_Oils. DOI: 10.3390/molecules200712016.

NOGUEIRA,T.F. Caracterização Genética e Química e Atividade Biológica do òleo Essencial de Populções Naturais de ELIONURUS MUTICUS HUMB \& BOMPL Ex WILLD. [s.I.]. Tese de doutorado. faculdade de Agronomia. Universidade Federal do Rio Grande do Sul, RS, BRASIL. 114, P. Junho, 2013. <Disponível em: https://www.lume.ufrgs.br/handle/10183/79635>.

RUFINO, M. S. M.; ALVES, R. E.; BRITO, E. S.; MORAIS, S. M.; SAMPAIO, C. G.;PEREZ-JIMÉNEZ, J.et al. Metodologia científica: Determinação da AtividadeAntioxidante Total em Frutas pelo Método de Redução do Ferro (FRAP).Comunicado Técnico Online, Embrapa 125. Fortaleza, CE, 4p, 2006. Disponível em: <http://www.cnpat.embrapa.br/cnpat/down/index.php?pub/cot_125.pdf>.

RUFINO, M. S. M.; ALVES, R. E.; BRITO, E. S.; MORAIS, S. M.; SAMPAIO, C. G.;PEREZ-JIMÉNEZ, J. et al. Metodologia científica: Determinação da Atividade Antioxidante Total em Frutas pela Captura do Radical Livre DPPH. Comunicado Técnico Online Embrapa 127. Fortaleza, CE, 4p, 2007. Disponível em:<http://www.cnpat.embrapa.br/cnpat/down/index.php?pub/Cot_127.pdf>.

SATYAL, P.; MURRAY, B. L.; MCFEETERS, R. L.; SETZER, W. N. Essential Oil Characterization of Thymus vulgaris from Various Geographical Locations. Foods, v. 5, n. $\quad 4, \quad$ p. $\quad 70, \quad 2016 . \quad$ Disponível em: <https://www.ncbi.nlm.nih.gov/pmc/articles/PMC5302419/>. DOI: 
10.3390/foods5040070.

SCHERER, R.; GODOY, H. T. Antioxidant activity index (AAI) by the 2,2-diphenyl-1picrylhydrazyl method. Food Chemistry, v.112, p.654-658, 2009. Disponível em: <https://www.sciencedirect.com/science/article/pii/S0308814608007218?casa_token =FRwDXwVmY14AAAAA:hyn7wbhjdUVjX6atsF6zp7BGK8LFX9Q8FkbfdPU1CZ16Z 44z4cl_h_PWT3LnuZ1Cr9bYBLU3An0.>DOI: 10.1016/j.foodchem.2008.06.026.

SILVA, C. B.; SILVA, K. B.; OLIVEIRA, E. L. S.; SOARES, V. F.; COSTA, J. G.; SANTOS, A. F. A importância da ação antioxidante de óleos essenciais em benefício da saúde. Diversitas Journal, v. 2, n. 1, p. 52, 2017. Disponivel em: $<$ https://diversitasjournal.com.br/diversitas_journal/article/view/483>.DOI: 10.17648/diversitas-journal-v2i4.483.

TORRAS, J.; GRAU, M. D.; LÓPEZ, J. F.; HERAS, F. X. C. Analysis of essential oils from chemotypes of Thymus vulgaris $L$ in Catalonia. Journal of the Science of Food and Agriculture, v. 87, n. 12, p. 2327-2333, 2007. Disponível em: <https://onlinelibrary.wiley.com/doi/epdf/10.1002/jsfa.2995>. DOI: 10.1002/jsfa.2995.

WESOLOWSKA, A.; JADCZAK, D. Comparison of the Chemical Composition of Essential Oils Isolated from Two Thyme (Thymus vulgaris L.) Cultivars. Notulae Botanicae Horti Agrobotanici Cluj-Napoca, v. 47, n. 3, p. 829-835, 2019. Disponível em: <https://www.notulaebotanicae.ro/index.php/nbha/article/view/11451>. DOI: doi.org/10.15835/nbha47311451. 